\title{
Superior Limbic Keratoconjunctivitis (SLK)
}

\author{
J. D. NELSON
}

Minnesota

\begin{abstract}
Summary
Superior limbic keratoconjunctivitis (SLK) is a disease characterised by inflammation of the upper palpebral and superior bulbar conjunctiva, keratinisation of the superior limbus and corneal and conjunctival filaments. It may be associated with other diseases such as keratoconjunctivitis sicca, hyperthyroidism and hyperparathyroidism. Although its exact aetiology is unknown, a mechanical hypothesis seems most attractive. Treatment is aimed at altering the abnormal mechanical interaction of the upper lid and superior corneal limbus.
\end{abstract}

Superior Limbic Keratoconjunctivitis is a disease of unknown aetiology which is characterised by

(1) inflammation of the superior tarsal conjunctiva;

(2) inflammation of the superior bulbar conjunctiva;

(3) fine punctate staining of the cornea adjacent to the superior corneal limbus;

(4) proliferation of superior limbic epithelial cells;

(5) filaments of the upper cornea and limbus. ${ }^{1}$

The disease was probably first noted in 1953 by Braley and Alexander who described patients with superficial punctate keratitis and filaments. ${ }^{2}$ The disease was further described by Thygeson in $1961^{3}$ in 12 patients with a filamentary keratitis limited to the superior cornea associated with inflammation of the superior bulbar conjunctiva. Thygeson and Kimura further elaborated on the disease in 1963. ${ }^{4}$ However, it was not until later in 1963 that Frederick H. Theodore gave the first complete description of the disease and sug- gested its present name, superior limbic keratoconjunctivitis (SLK). ${ }^{1}$

\section{Aetiology}

The exact cause of SLK is unknown, although infectious, ${ }^{4}$ immunologic, ${ }^{5}$ and mechanical aetiologies have been postulated. ${ }^{6}$ Keratoconjunctivits sicca has also been suggested as a cause. A viral aetiology is unlikely as viral cultures have been universally negative and no viral particles have been seen by transmission electron microscopy.? Although an autoimmune aetiology has been proposed due to the association of thyroid abnormalities with SLK,,$^{5.8}$ this is unlikely as there are minimal inflammatory changes seen by light microscopy, no immunoglobulin deposition by direct immunofluorescence, no circulating antibodies by indirect immunofluorescence, no eosinophils and the poor response to topical cortical steroids. ${ }^{9}$ Keratoconjunctivitis sicca (as defined by an abnormal Schirmer test) has been noted in approximately onequarter of patients with SLK ${ }^{10-13}$ (Table I).

Wright has proposed that the superior pal-

From Departments of Ophthalmology, St Paul-Ramsey Medical Center and Ramsey Clinic, St Paul, Minnesota and the University of Minnesota, Minneapolis, Minnesota.

Correspondence to: J. Daniel Nelson, MD, Department of Ophthalmology, St Paul-Ramsey Medical Center, St Paul, MN, 55101, USA. 
Table I Summary of clinical findings in patients with SLK

\begin{tabular}{|c|c|c|c|c|c|c|c|c|}
\hline & \multicolumn{8}{|c|}{ Author } \\
\hline & Corwin ${ }^{10}$ & Cher 5 & Wright & Passons"l & Udell ${ }^{12}$ & Ohashil3 & Nelson & Mean \% \\
\hline No. of patients & 22 & 10 & 23 & 10 & 11 & 12 & 5 & - \\
\hline Age $30-55$ years & 19 & 8 & —* & 8 & —* & 12 & 5 & 88.1 \\
\hline Female & 18 & 8 & 8 & 8 & 7 & 11 & 5 & 69.9 \\
\hline Bilateral & 一* & 5 & 19 & 9 & 3 & —* & 5 & 69.5 \\
\hline Filaments & 15 & 2 & 12 & 2 & -* & —* & 4 & 50.0 \\
\hline Thyroid Disease & —* & 5 & 6 & 5 & —* & 3 & $1 \dagger$ & 33.3 \\
\hline $\begin{array}{l}\text { Decreased Schirmer } \\
\text { test }\end{array}$ & 1 & 0 & $-^{*}$ & 4 & 6 & 5 & 51 & 24.2 \\
\hline
\end{tabular}

* Specific information as to these findings were not reported.

† One other patient had hyperparathyroidism due to a parathyroid adenoma.

pebral conjunctiva is responsible for initiating the pathological process which results in SLK. ${ }^{6} \mathrm{He}$ suggested that a chronically inflamed upper lid might cause a disturbance of the normal maturation of cells of the bulbar conjunctiva giving rise to the symptoms and signs of SLK. The difficulty with this hypothesis is that the amount of superior tarsal inflammation is relatively mild and it is difficult to identify whether the superior bulbar or palpebral conjunctiva is the initial site of inflammation. ${ }^{7}$ Donshik, et al., ${ }^{7}$ have suggested that the abnormality resides in the superior bulbar conjunctiva.

Ostler has proposed a mechanical hypothesis. ${ }^{14} \mathrm{He}$ suggests that the various findings of SLK can be explained by the movement of the upper eye lid which, due to a thyroid abnormality, exophthalmos, or specific or nonspecific chronic inflammation, is in tight apposition to the superior bulbar conjunctiva. The superior bulbar conjunctiva, which may be lax due to congenital or ageing processes, is subjected to constant abnormal movement as the eyelid opens and closes. This abnormal movement results in a chronic irritation of the bulbar conjunctiva due to mechanical factors. $\mathrm{He}$ also suggests that the papillary hypertrophy and cellular infiltration of the superior palpebral conjunctiva arise due to these mechanical factors. Supporting this hypothesis are the findings that removal, or thermal cauterisation, of the abnormal bulbar conjunctiva or treatment of the upper palpebral conjunctiva with silver nitrate result in improvement of the signs and symptoms of
SLK. At the present time, a mechanical aetiology seems most attractive.

\section{Clinical Features}

Patients with SLK usually complain of irritation, or a gritty sensation, redness and a mucoid discharge from the eye. Other symptoms include foreign body sensation, burning and photophobia. These symptoms tend to vary in severity, with time and activity, and, generally coincide with clinical signs. Discomfort is greatly increased by the presence of limbal or corneal filaments.

SLK has been reported to occur in all age groups but is more common in patients between the ages of 30-55 years and women are more frequently affected than men (Table I). This disease is usually bilateral but may occur unilaterally and asymmetrical involvement is not unusual. Tenzel ${ }^{8}$ first suggested an association of SLK and thyroid disease which was later confirmed by Cher. 5 Since then others have found thyroid disease in 20-50 per cent of patients with SLK (Table I).

The classical findings of SLK, as initially reported by Theodore, are listed in Table II. These findings can be divided into those of the superior palpebral conjunctiva, the superior bulbar and limbal conjunctiva and the cornea. There is a papillary reaction of the superior palpebral conjunctiva which consists of many small papillae (Fig. 1). The inferior palpebral conjunctiva is strikingly normal. The superior bulbar conjunctiva is hyperemic and thickened and may appear lusterous. The hyperaemia and thickening extend in a $10 \mathrm{~mm}$ arc 
Table II Classical findings in patients with $S L K^{1.3}$

Age: $20-67$ years

Sex incidence: Women $>$ men

Duration: 1-10 years

Prognosis: Eventually clears

Usually bilateral

Papillary reaction of superior palpebral conjunctiva

Superior bulbar conjunctival injection

Fine punctate staining of superior cornea and conjunctiva

Rose Bengal staining of superior cornea and conjunctiva

Proliferation of superior limbus to varying degrees

Superior corneal and conjunctival filaments

Decreased Schirmer test in some patients

Occasional corneal hypesthesia

Favourable response to 0.5 per cent silver nitrate in some cases

from the superior limbus centred at 12:00 o'clock toward the superior rectus muscle, and may stain with rose bengal, and less so with fluorescein or Alcian Blue (Fig. 2). Occasionally, a pseudomembrane ${ }^{1}$ or a subconjunctival haemorrhage may be seen. ${ }^{6}$ The superior one-third of the cornea is involved with a fine punctate keratopathy which stains with rose bengal and fluorescein. In about one-half of patients (Table I), multiple filaments may be found on the cornea, superior bulbar and palpebral conjunctiva (Fig. 3).

Decreased corneal sensation has been observed in one patient with many filaments, which returned to normal when the filaments disappeared.' Acquired 'with-the-rule' astigmatism which disappeared with resolution of SLK has been reported. ${ }^{5}$ Decreased tear secretion (as evidenced by an abnormal Schirmer test) may be seen in approximately one-quarter of SLK patients (Table I). Viral cultures are universally negative, although staphylococci have been isolated on bacterial cultures. ${ }^{1}$

The natural history of the disease involves a chronic, prolonged course with gradual, complete clearing. Short remissions are seen during the early course of the disease, which gradually lengthen until the disease disappears. Occasionally treatment of underlying thyroid disease, if present, will result in improvement of the signs and symptoms of SLK.

During 1984, five patients with SLK were seen in the Department of Ophthalmology at the University of Minnesota. Table III summarises the findings in these patients. Interestingly, one patient, who presented with classical SLK, was found to have a very elevated serum calcium and parathyroid hormone. Subsequently she was found to have a very large parathyroid adenoma. Surgical removal of the adenoma resulted in total resolution of her symptoms and clinical findings.

\section{Histology}

By light microscopy, biopsy specimens of superior bulbar conjunctival specimens show marked keratinisation of the epithelial cells and acanthosis 7.15 (Fig. 4). Some epithelial cells are swollen and in various stages of degeneration. Few goblet cells are present. ${ }^{6}$

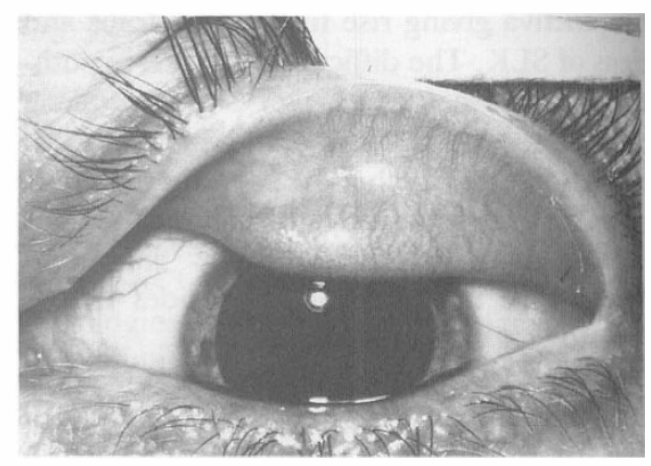

Fig. 1. The superior palpebral conjunctiva of a patient with SLK. Note the increased vascularity and the fine papillary reaction of the palpebral conjunctiva.

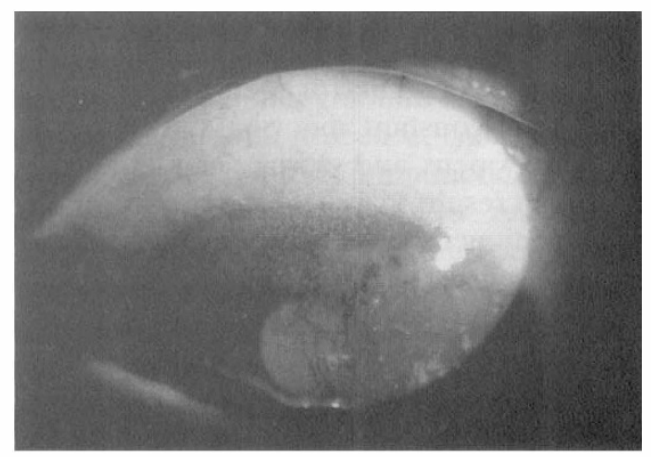

Fig. 2. Fluorescein staining of the superior limbus and bulbar conjunctiva of a patient with SLK. Note the fine punctate staining involving the superior cornea, limbus and conjunctiva. 


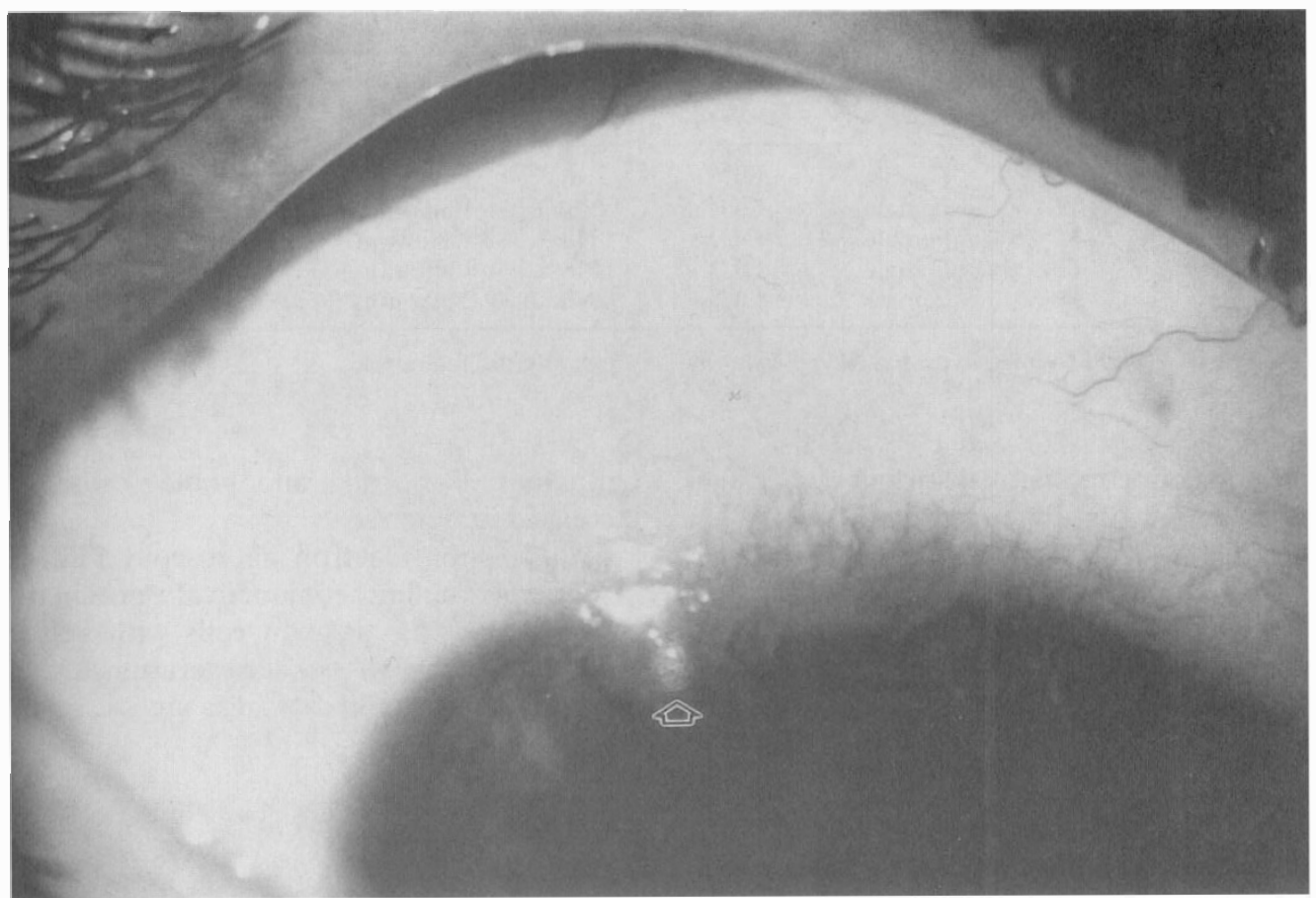

Fig. 3. The superior limbus of a patient with SLK demonstrating a corneal filament (arrow).

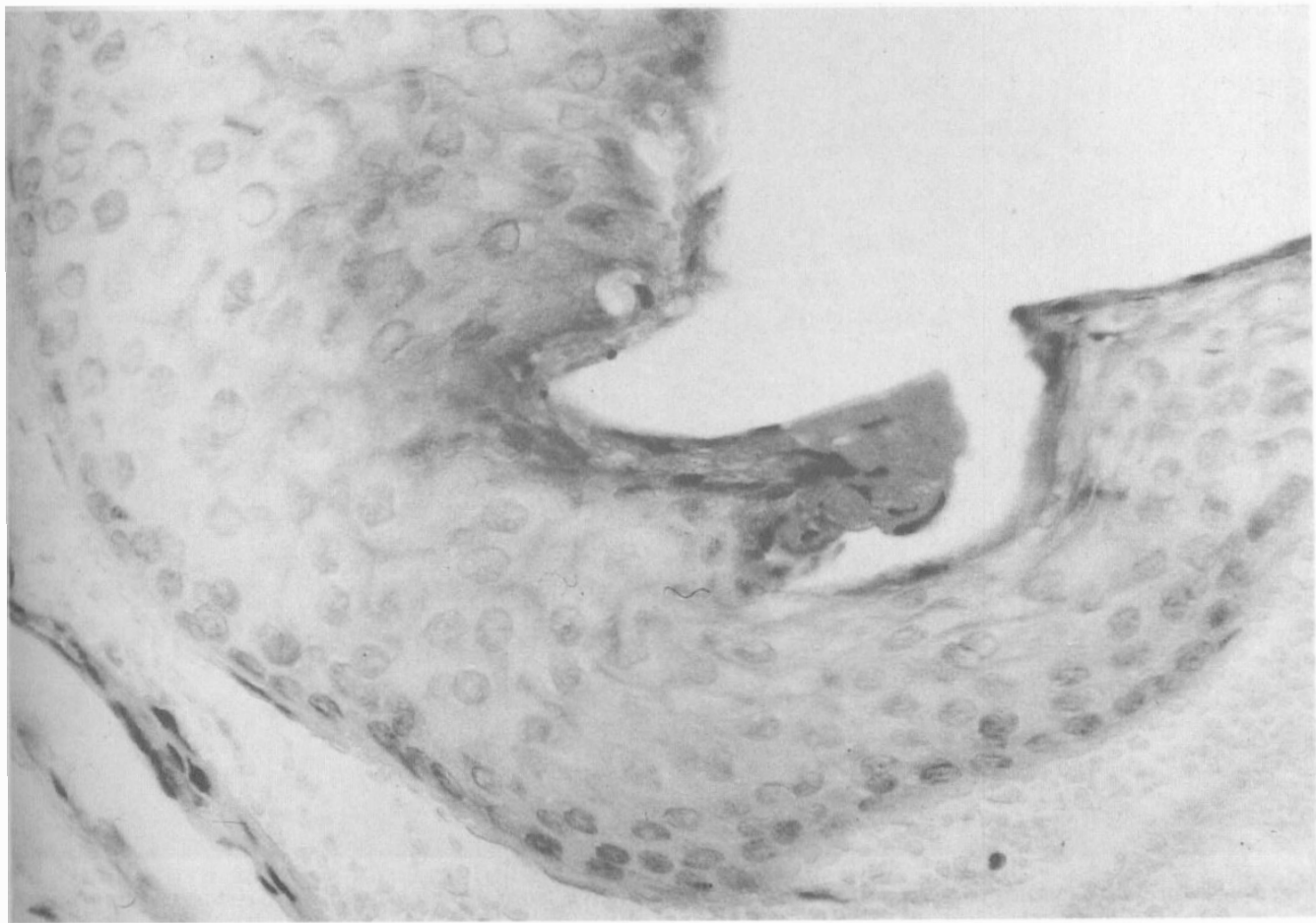

Fig. 4. A superior conjunctival biopsy specimen from a patient with $S L K$. Note the acanthosis and keratinisation of the epithelium with the absence of goblet cells. (Haematoxylin and eosin stain, original magnification $\times 50$.) 
Table III Summary of patients with SLK seen at the University of Minnesota during 1984

\begin{tabular}{cccccccc}
\hline Patient & $\begin{array}{c}\text { Age } \\
\text { (years) }\end{array}$ & Sex & $\begin{array}{c}\text { Eye } \\
\text { involved }\end{array}$ & $\begin{array}{c}\text { Hyper- } \\
\text { thyroidism }\end{array}$ & $\begin{array}{c}\text { Duraton } \\
\text { of symptoms }\end{array}$ & $\begin{array}{c}\text { Filaments } \\
\text { Schirmer test }\end{array}$ \\
\hline 1 & 32 & Female & OU & No & 4 months & Yes & No \\
2 & 35 & Female & OU & Yes & 18 months & Yes & Yes \\
3 & 44 & Female & OU & Not & Unknown & No & No \\
4 & 47 & Female & OU & No & 3 months & Yes & No \\
5 & 54 & Female & OU & No & 24 months & Yes & No \\
\hline
\end{tabular}

† Patient was found to have hyperparathyroidism due to a parathyroid adenoma.

The superior conjunctival stroma shows signs of oedema, with dilated lymphatics; very few inflammatory cells are present. ${ }^{7}$ By light microscopy, biopsy specimens of the superior tarsal conjunctiva shows infiltration of polymorphonuclear neutrophilic leukocytes (PMNs), lymphocytes and plasma cells. The epithelium is normal and goblet cells are increased in numbers. ${ }^{6}$

Transmission electron microscopy (TEM) of superior bulbar conjunctival specimens ${ }^{7}$ show large, pale, swollen cells with scarce organelles with balloon degeneration of the nuclei. The intracellular spaces are increased

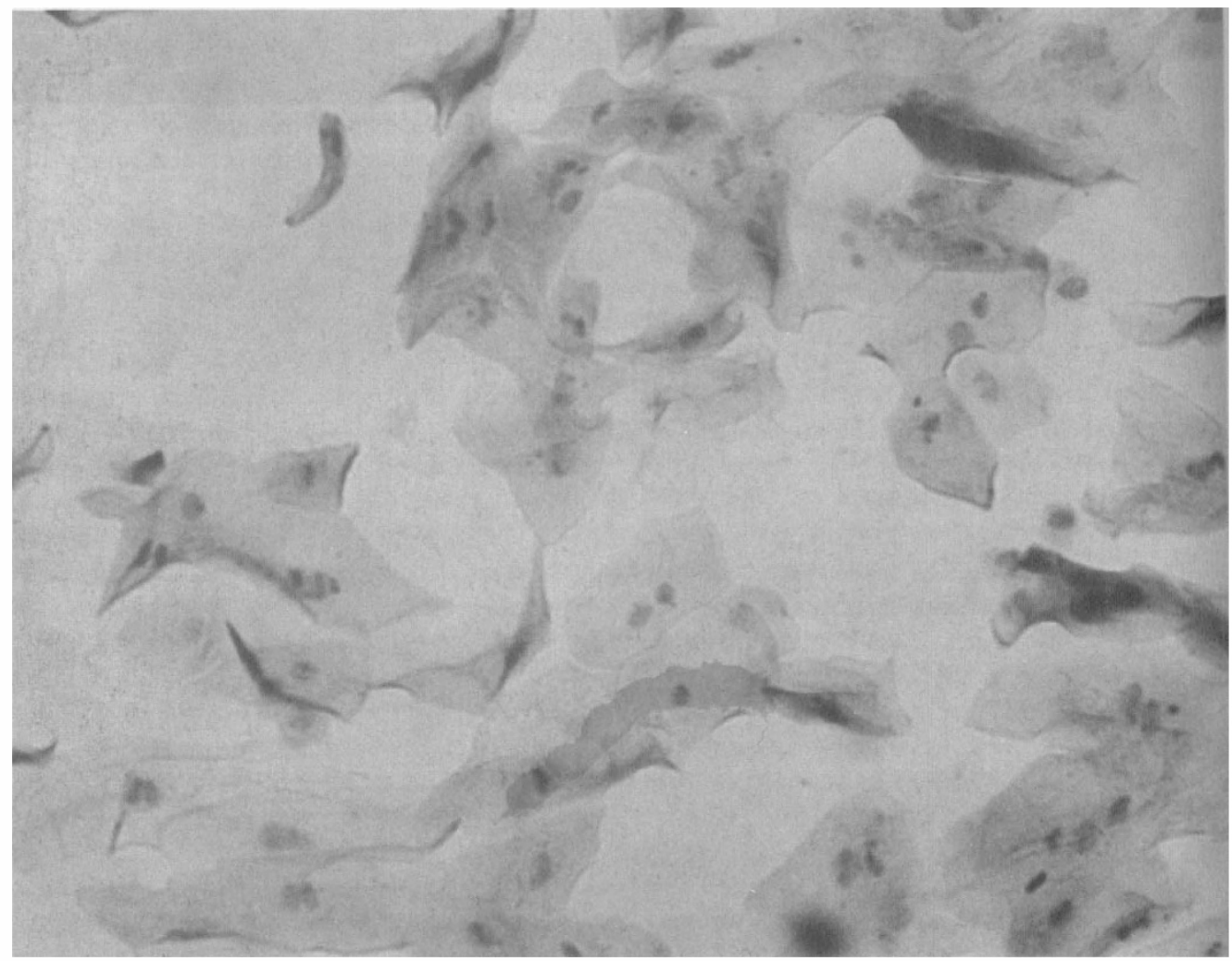

Fig. 5. Impression cytology of the superior bulbar conjunctiva from a patient with SLK demonstrating severe squamous metaplasia. The epithelial cells are very large and polygonal in shape. Some cells are keratinised and others are multi-nucleated. There are no goblet cells present. (Haematoxylin and PAS stain, original magnification $\times 50$.) 


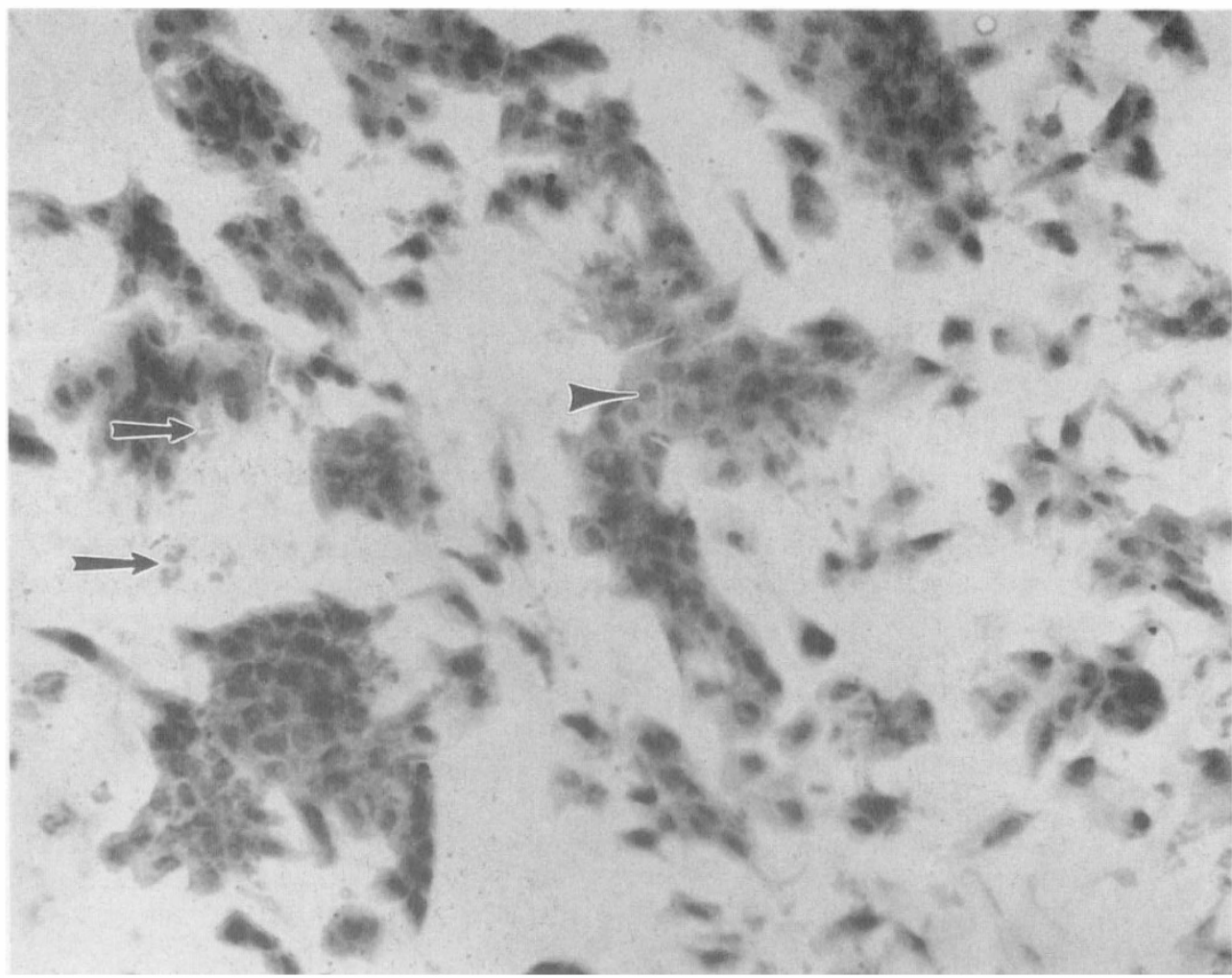

Fig. 6. Impression cytology of the superior palpebral conjunctiva from a patient with $S L K$. The epithelial cells are of normal size and shape (arrowhead). There are numerous inflammatory cells present (arrows). There are no goblet cells present in this specimen. (Haematoxylin and PAS stain, original magnification $\times 50$.)

with some breakdown of the desmosomes. There is extensive intracellular accumulation of glycogen granules. There is keratinisation of the epithelial cells with increased numbers and clumping of microfilaments, the presence of secondary lysosomes, thickening of the epithelial cellular membranes and the presence of keratohyaline granules in the cytoplasm.

\section{Diagnosis}

As previously mentioned, viral cultures of the conjunctiva in patients with SLK are negative and bacterial cultures show only growth of normal flora. Conjunctival scrapings of the superior bulbar conjunctiva show keratinised, hyalinised epithelial cells with PMNs and few lymphocytes. ${ }^{16}$ Scrapings of the superior tarsal conjunctiva show some keratinised epithelial cells and PMNs. Nuclei may show an unusual pattern of chromatin clumping (snake-like chromatin) with Papanicolaou stained scrapings. ${ }^{17}$ Impression cytology may provide useful information in patients with SLK. ${ }^{12.18}$ Interpalpebral and inferior palpebral specimens are usually normal, although occasionally mild-to-moderate degrees of squamous metaplasia may be seen on the interpalpebral specimens. Impressions of the superior bulbar conjunctiva show

Table IV Response of patients with SLK to various modalities of treatment

\begin{tabular}{lc}
\hline Treatment & $\begin{array}{c}\text { Resolution } \\
\text { or } \\
\text { improvement } \\
\text { (per cent) }\end{array}$ \\
\hline Conjunctival resection $^{11}$ & 80 \\
Thermocautery $^{12}$ & 73 \\
Vitamin A Topically $^{13}$ & 83 \\
Patching/bandage contact lenses $^{22}$ & 100 \\
n-Acetylcysteine $^{6}$ & 65 \\
\hline
\end{tabular}




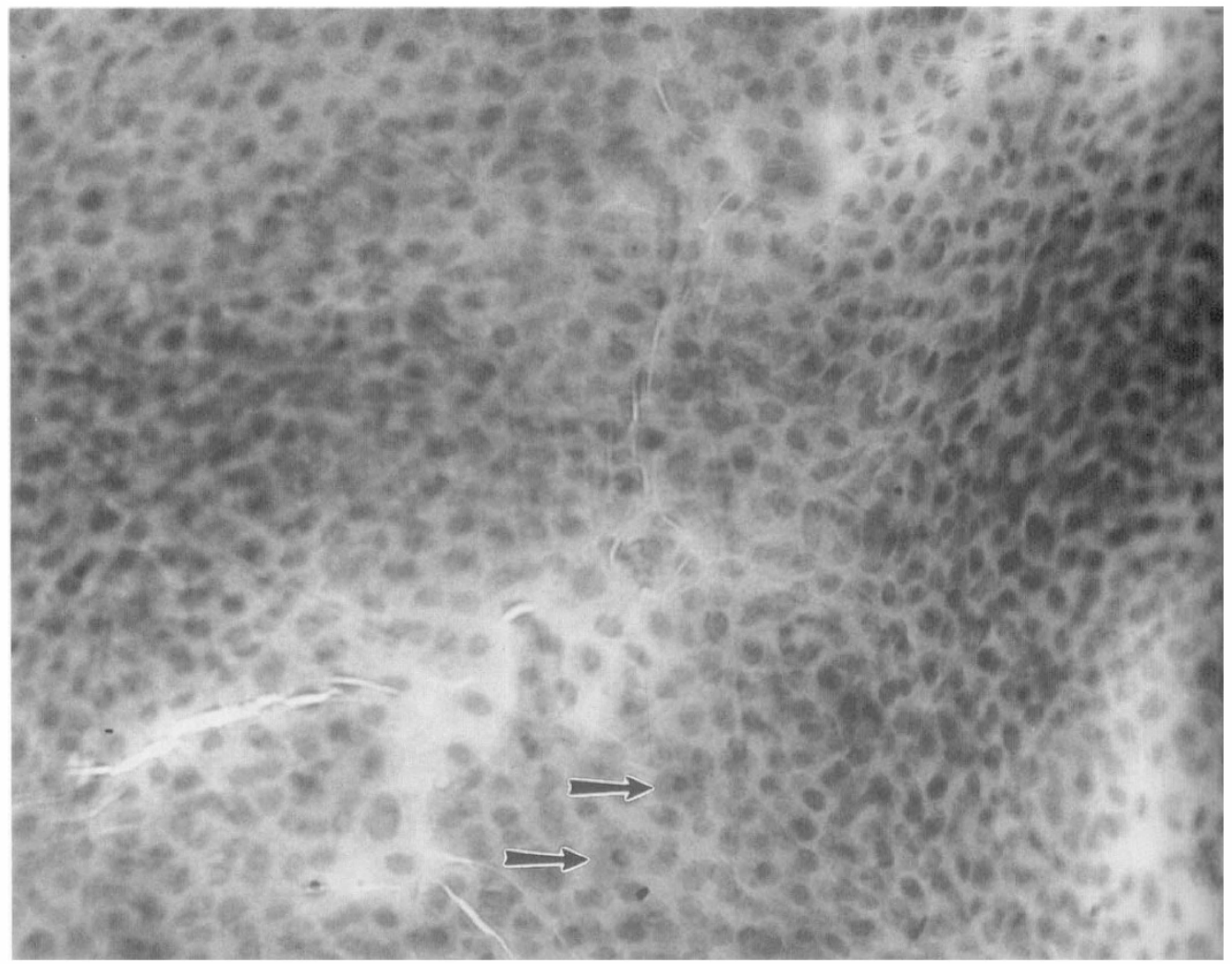

Fig. 7. Impression cytology of the interpalpebral bulbar conjunctiva from a patient with SLK. The epithelial cells are of normal size and shape. Goblet cells are present (arrows), but are difficult to demonstrate in this black-andwhite photomicrograph. (Haematoxylin and PAS stain, original magnification $\times 50$.)

severe squamous metaplasia with large irregularly-shaped epithelial cells, nuclear cytoplasmic ratios greater than $1: 6$, small, pyknotic nuclei and the absence of goblet cells (Fig. 5). Impressions of the superior palpebral conjunctiva show relatively mild squamous metaplasia, few goblet cells, and many inflammatory cells (Fig. 6).

An entity similar to SLK has been associated with contact lens wear. ${ }^{19-21}$ The clinical changes have been attributed to preservatives, especially thimerosal. ${ }^{22}$ Biopsy specimens of the superior bulbar conjunctiva ${ }^{19}$ show intracellular oedema, acanthosis, pseudoepitheliomatous hyperplasia, keratinisation, PMNs and plasma cells by light microscopy. TEM shows flattening of the microvilli and increased intracellular keratin filaments. Unlike SLK, keratinisation is not severe, nuclear degeneration is not present and there is no glycogen accumulation. These findings, along with clinical findings which show only minimal tarsal conjunctival involvement, usual absence of filaments, the presence in mostly younger females with no thyroid history and the worsening of symptoms and signs with contact lenses suggest that this condition is not classical SLK. In view of this, the term contact lens-induced SLK is not correct and the term contactlens-induced keratoconjunctivitis should be used.

\section{Treatment}

Many types of treatment have been used in patients with SLK with varying degrees of success (Table IV). The classical treatment for SLK has been the local application of $0.5-$ 1.0 per cent silver nitrate to the superior palpebral conjunctiva. ${ }^{1}$ This usually results in relief of symptoms for 4-6 weeks and may be 


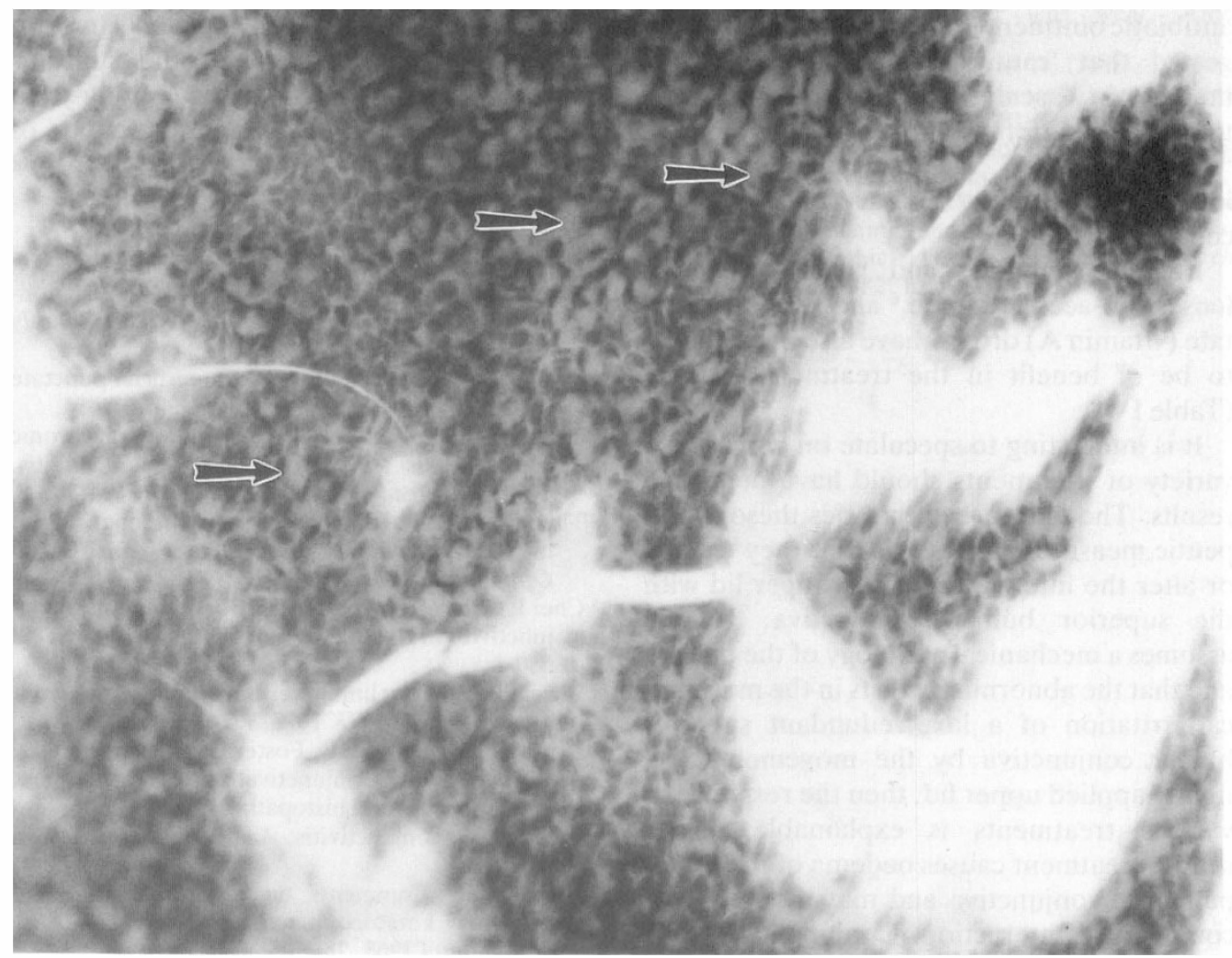

Fig. 8. Impression cytology of the inferior palpebral bulbar conjunctiva from a patient with $S L K$. The epithelial cells are of normal size and shape. Goblet cells are numerous (lighter stained cells, arrows). There are no inflammatory cells present. (Haematoxylin and PAS stain, original magnification $\times 50$.)

repeated every 4-6 weeks without any untoward effects. If the patient does not experience relief of symptoms after an initial application, the procedure may be repeated in 5-7 days. ${ }^{14}$ Silver nitrate is applied to the anaesthetised superior palpebral conjunctiva using a cotton-tipped applicator or cellulose sponge. As the silver nitrate can be slightly irritating to the eye itself, irrigation with sterile normal saline or balanced salt solution one minute after application is useful to prevent this. Cryotherapy may also be used but offers no advantages over silver nitrate therapy. ${ }^{14}$

Resection of the superior conjunctiva has been noted to be of benefit in patients who do not respond to treatment with silver nitrate..$^{7,11,23}$ Under local anaesthesia (topical and subconjunctival) the involved superior bulbar conjunctiva is dissected from Tenon's capsule and then resected along the limbus from 10 to 2 o'clock." Usually, $5-8 \mathrm{~mm}$ of conjunctiva are removed. The eye is patched for comfort overnight and antibiotic drops or ointments are used for an additional 48-72 hours. Those patients with SLK who have decreased Schirmer tests seem not to respond to conjunctival resection as well as those with normal Schirmer tests. "

Recently, thermocauterisation of the superior bulbar conjunctiva has been used to treat patients with SLK. ${ }^{17}$ Under infiltrative local anaesthesia, a disposable microsurgery cautery is used to apply 30 to 50 brief, focal applications to the superior bulbar conjunctiva between the 10 and 2 o'clock positions from the limbus to $8 \mathrm{~mm}$ posteriorly. Each burn should be of sufficient intensity to involve the entire epithelial layer and produce stromal shrinkage without injurying the underlying sclera. Post-operatively, a topical 
antibiotic ointment is applied. It has been suggested that 'cautery produces a transient increase in vascularity and epithelial migration and/or differentiation from surrounding "normal tissue," thereby disrupting the inflammatory cycle and restoring goblet cells. $^{12}$

Pressure patching and bandage contact lenses, ${ }^{24} \mathrm{n}$-acetylcysteine ${ }^{6}$ and retinol palmitate (vitamin A) drops ${ }^{13}$ have also been shown to be of benefit in the treatment of SLK (Table IV).

It is interesting to speculate on why such a variety of treatments should have beneficial results. The one thread that ties these therapeutic measures together is that they change or alter the interaction of the upper lid with the superior bulbar conjunctiva. If one assumes a mechanical aetiology of the disease and that the abnormality rests in the mechanical irritation of a lax, redundant superior bulbar conjunctiva by the movement of a tightly applied upper lid, then the response to various treatments is explainable. Silver nitrate treatment causes oedema of the upper palpebral conjunctiva and may alter the lidconjunctival interaction. Conjunctival resection removes the abnormally lax conjunctiva. Thermocauterisation of the superior conjunctiva tightens, or shrinks, the conjunctiva, removing the abnormal laxity. Pressure patching relieves the lid-limbus interaction and bandage contact lenses protect the limbus from the action of the upper lid. Various types of topical medications may reduce the trauma to the limbus by the lid by providing lubrication.

If a mechanical aetiology is, indeed, responsible for SLK, there is probably not an easy way to prevent this relatively uncommon disease. However, once the disease presents with symptoms and findings, treatment should be aimed at resolving the abnormal lidsuperior conjunctival interaction. The use of lubricants, topical vitamin A or n-acetylcysteine is a reasonable initial choice of treatment. If an adequate response is not obtained, application of silver nitrate or pressure patching may be tried. Inprovement is usually temporary and often has to be repeated on an ongoing basis. Placement of a bandage contact lens will result in resolution of symptoms as long as the lens remains in place. Thermocauterisation or resection of the superior bulbar conjunctiva may provide long-term improvement in the signs and symptoms of SLK in unresponsive cases.

\section{References}

1 Théodore FH: Superior Limbic Keratoconjunctivitis. Eye, Ear, Nose and Throat Monthly 1963, 42: 25-8.

${ }^{2}$ Braley AE and Alexander RC: Superficial punctate keratitis. Arch Ophthalmol 1953, 50: 147-54.

${ }^{3}$ Thygeson $\mathrm{P}$ and Kimura SJ: Observations on chronic conjunctivitis and chronic keratoconjunctivitis. Trans Am Acad Ophthalmol 1963, 67: 494-17.

4 Theodore FH: Further observations on superior limbic keratoconjunctivitis. Trans Am Acad Ophthalmol Otolaryngol 1967, 71: 341-51.

${ }^{5}$ Cher I: Clinical features of superior limbic keratoconjunctivitis in Australia. Arch Ophthalmol 1969, 82: 580-6.

${ }^{6}$ Wright P: Superior limbic keratoconjunctivitis. Trans Ophthalmol Soc UK 1972, 92: 555-60.

${ }^{7}$ Donshik PC, Collin HB, Foster CS, Cavanagh HD, Boruchoff SA: Conjunctival resection treatment and ultrastructural histopathology of superior limbic keratoconjunctivitis. Am J Ophthalmol 1978, 85: 101-10.

${ }^{8}$ Tenzel RR: Comments on superior limbic filamentous keratoconjunctivitis: Part 2. Arch Ophthalmol 1968. 78: 505.

${ }^{9}$ Eiferman RA and Wilkins EL: Immunological aspects of superior limbic keratoconjunctivitis. Canad J Ophthalmol 1979, 14: 85-7.

1" Corwin ME: Superior limbic keratoconjunctivitis. Am J Ophthalmol 1968, 66: 338-40.

"Passons GA and Wood TO: Conjunctival resection for superior limbic keratoconjunctivitis. Ophthalmology 1986, 91: 966-8.

12 Udell IJ, Kenyon KR, Sawa M: Treatment of superior limbic keratoconjunctivitis by thermocauterisation of the superior bulbar conjunctiva. Ophthalmology 1986, 93: 162-66.

${ }_{13}^{3}$ Ohashi Y, Watanabe H, Kinoshita S, et al.: Vitamin A eye drops for superior limbic keratoconjunctivitis. Am J Ophthalmol 1988, 105: 523-7.

${ }_{14}$ Ostler HB: Superior limbic keratoconjunctivitis. In Smolin G, Thoft RA, eds. The Cornea, Boston/ Toronto: Little Brown 1987: 296-98.

15 Collin HB, Donshik PC, Foster CS, Boruchoff SA: Keratinisation of the superior bulbar conjunctival epithelium in superior limbic keratoconjunctivitis in humans. Acta Ophthalmol 1978, 56: 531-43.

16 Theodore FH and Ferry AP: Superior limbic keratoconjunctivitis: Clinical and pathological correlations. Arch Ophthalmol 1970, 84: 481-4.

${ }_{17}$ Wander AH and Masukawa T: Unusual appearance of condensed chromatin in conjunctival cells in superior limbic keratoconjunctivitis. Lancet 1981, ii: $42-3$.

${ }^{18}$ Nelson JD and Wright JC: Goblet cell densities in 
ocular surface disease. Arch Ophthalmol 1984, 102: 1049-51.

${ }^{19}$ Sendele DD, Kenyon KR, Mobilia EF, et al.: Superior limbic keratoconjunctivitis in contact lens wear. Ophthalmology 1983, 90: 617-22.

${ }^{20}$ Fuerst DJ, Sugar J, Worobec S: Superior limbic keratoconjunctivitis associated with cosmetic soft contact lens wear. Arch Ophthalmol 1983, 101: 1214-16.

${ }^{21}$ Stenson S: Superior limbic keratoconjunctivitis asso- ciated with soft contact lens wear. Arch Ophthalmol 1983, 101: 402-4.

22 Wilson LA, McNatt J, Reitschel R: Delayed hypersensitivity to thimerosal in soft contact lens wearers. Ophthalmology 1981, 88: 804-9.

${ }^{23}$ Tenzel RR: Resistant superior limbic keratoconjunctivitis. Arch Ophthalmol 1973. 89: 439.

${ }^{24}$ Mondino BJ, Zaidman GW, Salamon SW: Use of pressure patching and soft contact lenses in superior limbic keratoconjunctivitis. Arch Ophthalmol 1982, 100: 1932-4. 